\title{
Prevalência de lesões psicossomáticas na cavidade bucal em policiais militares do
}

\section{Estado de Alagoas}

\author{
Prevalence of psychosomatic lesions in the oral cavity in police officers from the State of Alagoas \\ Prevalencia de lesiones psicosomáticas en la cavidad bucal en policías del Estado de Alagoas
}

Iris Marilia Alves da Silva

ORCID: https://orcid.org/0000-0001-396-213X

Universidade Federal de Alagoas, Brasil

E-mail: iris.silva@foufal.ufal.br

Érika Priscila Santos Melo

ORCID: https://orcid.org/0000-0003-1102-2208

Universidade Federal de Alagoas, Brasil

E-mail: erika.melo@foufal.ufal.br

Larissa de Oliveira Carvalho Borges

ORCID: https://orcid.org/0000-0002-5389-5528

Universidade Federal de Alagoas, Brasil

E-mail: larissaocborges@gmail.com

Josimeire Barbosa Silva

ORCID: https://orcid.org/0000-0002-7173-1156

Centro Universitário CESMAC, Brasil

E-mail: meirissima2020@ hotmail.com

Luiz Carlos Oliveira dos Santos

ORCID: https://orcid.org/0000-0002-7021-5491

Universidade Federal de Alagoas, Brasil

E-mail: luiz.santos@foufal.ufal.br

\begin{abstract}
Resumo
O presente artigo aborda um levantamento da prevalência de doenças psicossomáticas com repercussão na cavidade bucal em indivíduos que demandam da Polícia Militar de Alagoas (PMAL). A pesquisa teve como objetivo identificar a frequência destas lesões em policiais militares pertencentes ao $1^{\circ}$ Batalhão da PMAL, relacionando-as a fatores como faixa etária, gênero, atividade profissional e patente. A hipótese levantada foi de que o estresse no trabalho pode contribuir para o surgimento de lesões bucais psicossomáticas nos policiais da PMAL. Trata-se de um estudo transversal analítico com abordagem quantitativa e qualitativa em pacientes que demandam do $1^{\circ}$ Batalhão da Polícia Militar de Alagoas. A metodologia empregada baseou-se em entrevista seguida de exame clínico da cavidade bucal em cada um dos policiais incluídos na pesquisa e fundamentou-se em uma revisão bibliográfica acerca do assunto. Foram detectados através dos exames e relatos dos pacientes casos de ulceração aftosa recorrente, herpes labial, bruxismo, xerostomia, mucosa mordiscata e língua geográfica. Os estudos apontam uma prevalência de lesões similar ao observado na população em geral, não havendo diferenças significativas com relação a gênero, faixa etária, patente e atividade profissional. Ressalta-se a importância do correto diagnóstico de lesões na cavidade bucal com o propósito de orientar, tratar e proporcionar melhor qualidade de vida aos pacientes.
\end{abstract}

Palavras-chave: Diagnóstico bucal; Estresse ocupacional; Medicina psicossomática.

\begin{abstract}
This article addresses a survey of the prevalence of psychosomatic diseases with repercussions in the oral cavity in individuals who demand from the Military Police of Alagoas (PMAL). The research aimed to identify the frequency of these injuries in military police officers belonging to the 1st Battalion of PMAL, relating them to factors such as age group, gender, professional activity and rank. The hypothesis raised was that stress at work may contribute to the emergence of psychosomatic oral lesions in PMAL police officers. This is a cross-sectional analytical study with a quantitative and qualitative approach in patients who demand from the 1st Battalion of the Military Police of Alagoas. The methodology used was based on an interview followed by a clinical examination of the oral cavity in each of the policemen included in the research and was based on a bibliographic review on the subject. Cases of recurrent aphthous ulceration, cold sores, bruxism, xerostomia, nibbling mucosa and geographical tongue were detected through the exams and reports of the patients. Studies show a prevalence of injuries similar to that observed in the general population, with no significant differences in relation to gender, age group, patent and professional activity. The importance of the correct diagnosis of lesions in the oral cavity is emphasized in order to guide, treat and provide a better quality of life to patients.
\end{abstract}


Keywords: Oral diagnosis; Occupational stress; Psychosomatic medicine.

\section{Resumen}

Este artículo aborda una encuesta sobre la prevalencia de enfermedades psicosomáticas con repercusión en la cavidad bucal en individuos que demandan a la Policía Militar de Alagoas (PMAL). La investigación tuvo como objetivo identificar la frecuencia de estas lesiones en policías militares pertenecientes al 1er Batallón del PMAL, relacionándolas con factores como grupo de edad, sexo, actividad profesional y rango. La hipótesis planteada fue que el estrés en el trabajo puede contribuir a la aparición de lesiones orales psicosomáticas en los agentes de policía de PMAL. Se trata de un estudio analítico transversal con abordaje cuantitativo y cualitativo en pacientes que demandan del 1er Batallón de la Policía Militar de Alagoas. La metodología utilizada se basó en una entrevista seguida de un examen clínico de la cavidad bucal en cada uno de los policías incluidos en la investigación y se basó en una revisión bibliográfica sobre el tema. A través de los exámenes e informes de los pacientes se detectaron casos de ulceración aftosa recurrente, herpes labial, bruxismo, xerostomía, mucosa mordisqueante y lengua geográfica. Los estudios muestran una prevalencia de lesiones similar a la observada en la población general, sin diferencias significativas en relación al sexo, grupo de edad, patente y actividad profesional. Se enfatiza la importancia del correcto diagnóstico de las lesiones en la cavidad bucal para orientar, tratar y brindar una mejor calidad de vida a los pacientes.

Palabras clave: Diagnóstico oral; Estrés laboral; Medicina psicosomática.

\section{Introdução}

A ideia de que o nosso corpo pode adoecer em consequência de problemas emocionais é um assunto que ganha cada vez mais espaço nos estudos contemporâneos. É evidente a forma como os fatores da organização do trabalho estão relacionados com o aparecimento dos sintomas psicossomáticos (Barbosa et al., 2020; Rabelo et al.,2018).

As emoções que afetam o indivíduo no ambiente de trabalho decorrentes do choque com a organização ocupacional e que podem gerar doenças, vem a ser definidas como doenças psicossomáticas (Carvalho, 2016). A palavra psicossomática é um termo tirado de psique (denotando mente, processos mentais, e atividades emocionais) e somático (soma, significando corpo e algo distinto da mente). A visão psicossomática aborda a doença não como um fato isolado no organismo, mas como resultado de um processo vivenciado pelo indivíduo (Silva et al., 2017; Reis \& Godinho, 2018).

Considera-se a profissão do policial militar como uma das mais estressantes na atualidade, todavia, seu serviço representa um notável recurso do Estado para a proteção do patrimônio público. Diante disso, tem sido recorrente investigações acerca do exercício da profissão, destacando as condições de trabalho, estresse ocupacional e sofrimento psíquico em policiais (Pelegrini et al., 2018; Santos et al., 2019).

Cruz et al., (2008) estudaram as seguintes condições bucais, relacionando-as ao estresse: líquen plano, língua geográfica, síndrome da boca ardente, ulceração aftosa recorrente e herpes simples recidivante. No presente trabalho, além das doenças citadas, também será avaliada a presença ou não do bruxismo e da mucosa mordiscata em um grupo de militares, por se tratar de uma categoria profissional favorável ao desenvolvimento de hábitos parafuncionais (Sarrazin \& Maia, 2020; Reche, 2018).

As doenças psicossomáticas são reflexos comuns de perturbações mentais, podendo, o profissional menos avisado, elaborar um diagnóstico errôneo ou que acarretará em problemas na definição da terapêutica (Alves e Lima, 2016). O presente trabalho pode trazer esclarecimentos aos profissionais da área a respeito destas afecções. É de relevância da mesma forma para os pacientes, que serão diagnosticados e acompanhados de acordo com o tipo da doença apresentada. Diante do exposto, questionou-se: qual a prevalência de lesões bucais psicossomáticas em militares da PMAL? Essa questão central da pesquisa desdobrou-se em outros questionamentos secundários, tais como: Há variações na prevalência com relação à idade, ao gênero, à atividade profissional e à patente? A hipótese levantada foi de que o estresse no trabalho pode contribuir para o surgimento de lesões bucais psicossomáticas nos policiais militares da PMAL.

O objetivo do estudo consistiu, dessa forma, em identificar a frequência destas lesões em policiais militares pertencentes ao $1^{\circ}$ Batalhão da PMAL, relacionando-as a fatores como faixa etária, gênero, atividade profissional e patente. A fundamentação teórica foi realizada através de revisão bibliográfica. 


\section{Metodologia}

Foi realizado um estudo transversal analítico com abordagem quantitativa e qualitativa (Costa, 2015). A população da pesquisa foi constituída de pacientes que demandam do $1^{\circ}$ Batalhão da Polícia Militar de Alagoas no período de julho a agosto de 2015, com propósito de delinear a frequência de lesões bucais relacionadas ao estresse.

$\mathrm{O}$ universo de estudo abrangeu os policiais pertencentes ao $1^{\circ} \mathrm{BPM}$, pois o mesmo foi objeto de pesquisa realizada pelo Centro de Assistência Social (CAS) da PMAL onde foi constatado alto nível de vulnerabilidade ao estresse. Esta pesquisa, aplicada em 2012, utilizou o teste psicológico EVENT (Escala de Vulnerabilidade ao Estresse no Trabalho) para avaliar o nível de vulnerabilidade ao estresse no trabalho.

Os critérios de inclusão no presente estudo foram: ser componente do $1^{\circ}$ Batalhão da PMAL, em atividade, ambos os gêneros, e aceitar participar da pesquisa assinando o termo de consentimento. Os critérios de exclusão foram: componentes não pertencentes ao $1^{\circ} \mathrm{BPM}$, componentes pertencentes ao $1^{\circ} \mathrm{BPM}$ inativos no momento da pesquisa.

Esses pacientes foram entrevistados e examinados individualmente: no primeiro momento foi utilizado um questionário individual com as variáveis relacionadas à idade, gênero, estado civil, cor, patente, nível de instrução, hábitos (tabagismo e etilismo), medicações utilizadas tempo da sintomatologia inicial da doença, tempo de evolução e recidiva da doença. No segundo momento realizou-se um exame clínico observando e identificando as lesões na cavidade bucal. Para esse procedimento foram utilizados os equipamentos de biossegurança (luva, gorro, máscara, jaleco), lanterna, abaixadores de língua e gaze.

Esse estudo foi aprovado pelo Comitê de Ética em Pesquisa da Universidade Federal de Alagoas - UFAL, obedecendo a Resolução no 466/2012 da Comissão Nacional de Ética em Pesquisa e em consonância com a Declaração de Helsinki. Após assinar o termo de livre consentimento, os pacientes responderam ao questionário proposto, e a etapa seguinte correspondeu ao exame clínico realizado pelo cirurgião-dentista responsável pela pesquisa.

As informações coletadas foram transferidas para um banco de dados e realizou-se uma avaliação percentual e estatística para apurar o resultado. Os resultados estão apresentados sob a forma de tabelas e gráficos formatados no Excel. A análise estatística foi realizada pelo qui quadrado e análise de variância no nível de significância de 0,05 . O programa utilizado foi o EpiInfo 3.5.4.

\section{Resultados e Discussão}

Primeiramente traçou-se um perfil do batalhão analisado, conforme Tabela 1.

O gênero masculino representou $88,4 \%$ da amostra total. Houve a prevalência da faixa etária entre 40-49 anos, havendo ainda 13,4\% com 50 anos ou mais. A idade média foi de 40 anos, com desvio padrão de 8,74.

Em se tratando da atividade profissional a maior parte exercia apenas função operacional, enquanto 6,3\% exerciam atividades operacionais e administrativas.

Os soldados corresponderam a 40,2\% da amostra, seguidos pelos cabos (30,4\%), sargentos $(24,1 \%)$, e 4,5\% eram oficiais. A maioria possuía ensino médio completo. Um pequeno percentual possuía pós-graduação $(4,5 \%)$. Os casados representaram maior percentual $(67,9 \%)$ em relação aos solteiros, divorciados, viúvos e consensuais. A renda familiar mensal acima de quatro salários mínimos prevaleceu (50,9\%), seguida por três salários $(26,8 \%)$, quatro salários $(18,8 \%)$ e dois ou menos $(2,7 \%)$. Os entrevistados em sua maioria $(74,1 \%)$ possuíam casa própria. 
Research, Society and Development, v. 10, n. 5, e22710514830, 2021

(CC BY 4.0) | ISSN 2525-3409 | DOI: http://dx.doi.org/10.33448/rsd-v10i5.14830

Tabela 1 - Perfil do $1^{\circ} \mathrm{BPM}-\mathrm{PMAL}-2015$

\begin{tabular}{|c|c|c|c|c|}
\hline \multirow[b]{2}{*}{ Variáveis } & \multirow[b]{2}{*}{$\mathbf{N}$} & \multirow[b]{2}{*}{$\%$} & \multirow{2}{*}{$\begin{array}{l}\text { Ic95\% } \\
\text { L. inf } \\
\end{array}$} & \multirow[b]{2}{*}{ L. sup } \\
\hline & & & & \\
\hline Gênero masculino & 99 & 88,4 & 81,0 & 93,7 \\
\hline Gênero feminino & 13 & 11,6 & 6,3 & 19 \\
\hline Faixa etária 20 a 29 & 21 & 18,8 & 12,0 & 27,2 \\
\hline Faixa etária 30 a 39 & 28 & 25,0 & 17,3 & 34,1 \\
\hline Faixa etária 40 a 49 & 48 & 42,9 & 33,5 & 52,6 \\
\hline Leucoderma & 56 & 50,0 & 40,4 & 59,6 \\
\hline Melanoderma & 56 & 50,0 & 40,4 & 59,6 \\
\hline Ensino médio completo & 66 & 58,9 & 49,2 & 68,1 \\
\hline Superior incompleto & 18 & 16,1 & 9,8 & 24,2 \\
\hline Superior completo & 23 & 20,5 & 13,5 & 29,2 \\
\hline Soldado & 45 & 40,2 & 31,0 & 49,9 \\
\hline Cabo & 34 & 30,4 & 22,0 & 39,8 \\
\hline Sargento & 27 & 24,1 & 16,5 & 33,1 \\
\hline Administrativo & 23 & 20,5 & 13,5 & 29,2 \\
\hline Operacional & 82 & 73,2 & 64,0 & 81,1 \\
\hline Solteiro & 23 & 20,5 & 13,5 & 29,2 \\
\hline Casado & 76 & 67,9 & 58,4 & 76,4 \\
\hline Casa própria & 83 & 74,1 & 65,0 & 81,9 \\
\hline Casa alugada & 25 & 22,3 & 15,0 & 31,2 \\
\hline Renda familiar 3 salários & 30 & 26,8 & 18,9 & 36,0 \\
\hline Renda familiar 4 salários & 21 & 18,8 & 12,0 & 27,2 \\
\hline Renda familiar $>4$ salários & 57 & 50,9 & 41,3 & 60,5 \\
\hline
\end{tabular}

Fonte: Autores.

Com relação às afecções bucais psicossomáticas detectadas, $38 \%$ da amostra apresentou pelo menos uma destas e $62 \%$ não apresentou nenhuma (Gráfico 1).

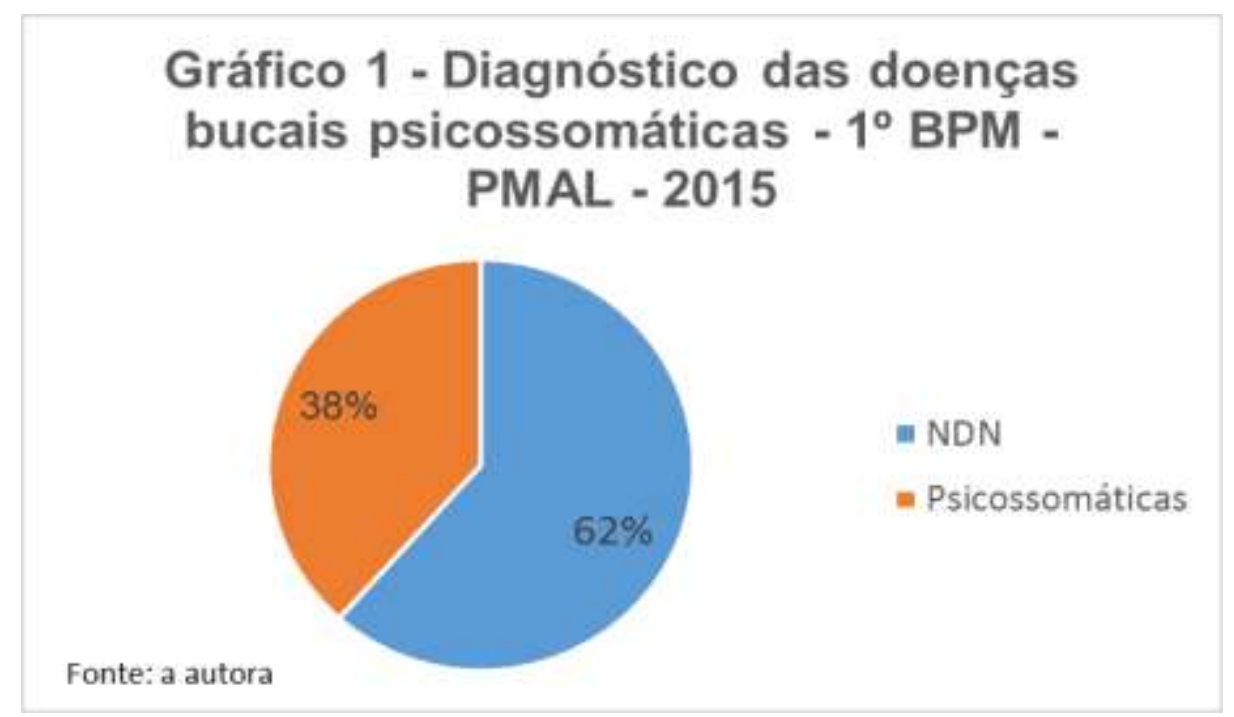


Foram encontradas ou relatadas as seguintes condições: língua geográfica, mucosa mordiscata, xerostomia, herpes labial, bruxismo e ulceração aftosa recorrente (Gráfico 2). Não houve diferenças estatisticamente significativas na prevalência das lesões com relação ao gênero, idade, cor, grau de instrução, patente, ocupação, situação conjugal e renda familiar.

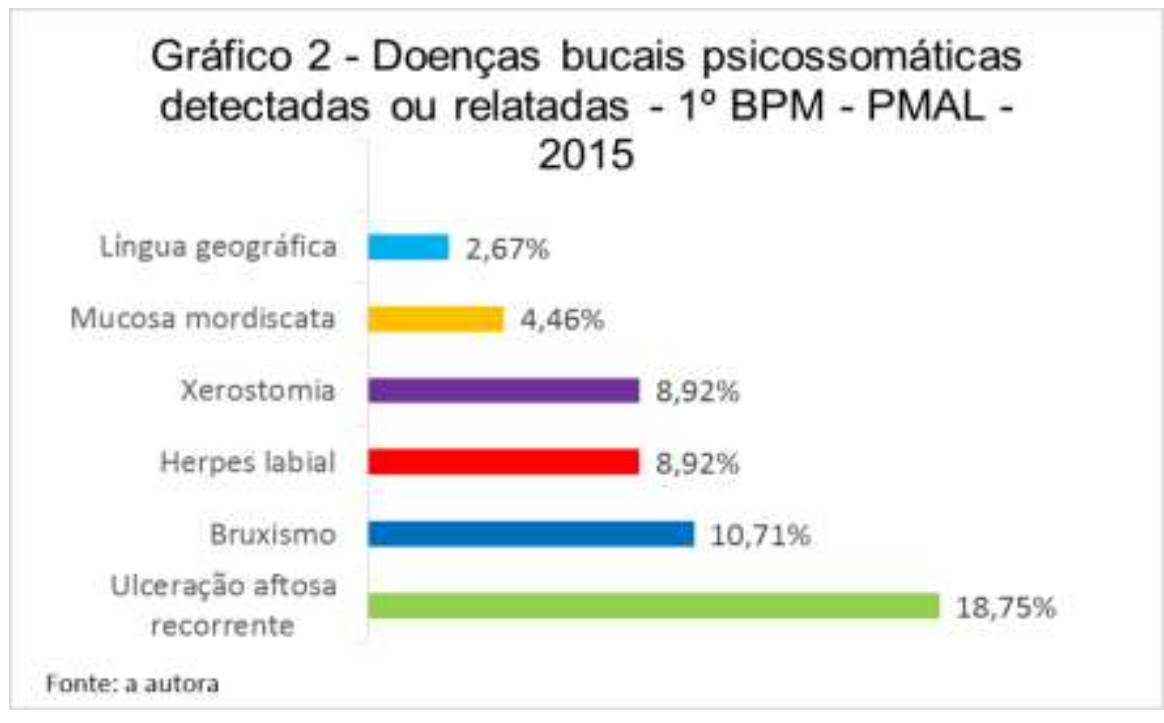

A ulceração aftosa recorrente foi a condição mais prevalente. Foram diagnosticados clinicamente dois casos (um destes evidenciado na Figura 1) e 19 entrevistados relataram sua ocorrência com alguma frequência. A prevalência encontrada (18,75\% da amostra) se aproxima da média da população em geral, que corresponde a 20\% segundo Neville et al., (2004).

Figura 1 - Ulceração aftosa recorrente em paciente do $1^{\circ} \mathrm{BPM}$ - PMAL - 2015.

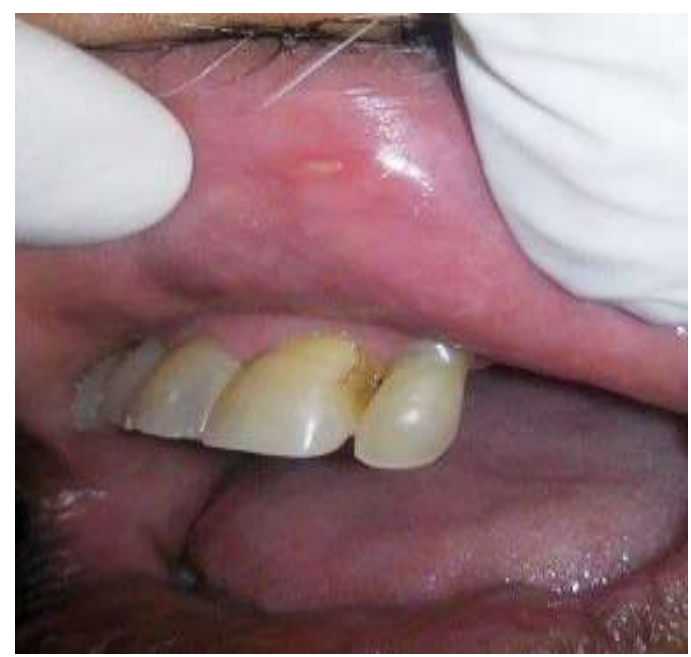

Fonte: Autores.

A Figura 1 mostra uma ulceração aftosa recorrente em mucosa de lábio superior, apresentando o característico formato circular com centro necrótico e superfície rasa, bordas elevadas e halo eritematoso.

O bruxismo foi detectado em 10,71\% da amostra (um caso é demonstrado nas Figuras 2 e 3). Nenhuma mulher foi diagnosticada com bruxismo. Porém, devido à minoria numérica das mesmas, este dado não foi significativo. De acordo com Carvalho et al., (2008) a literatura apresenta valores que variam de $6 \%$ a $90 \%$. Os mesmos autores encontraram 33,3\% de bruxistas em policiais militares do Maranhão. 
Figura 2 - Bruxismo em paciente do $1^{\circ} \mathrm{BPM}$ - PMAL - 2015.

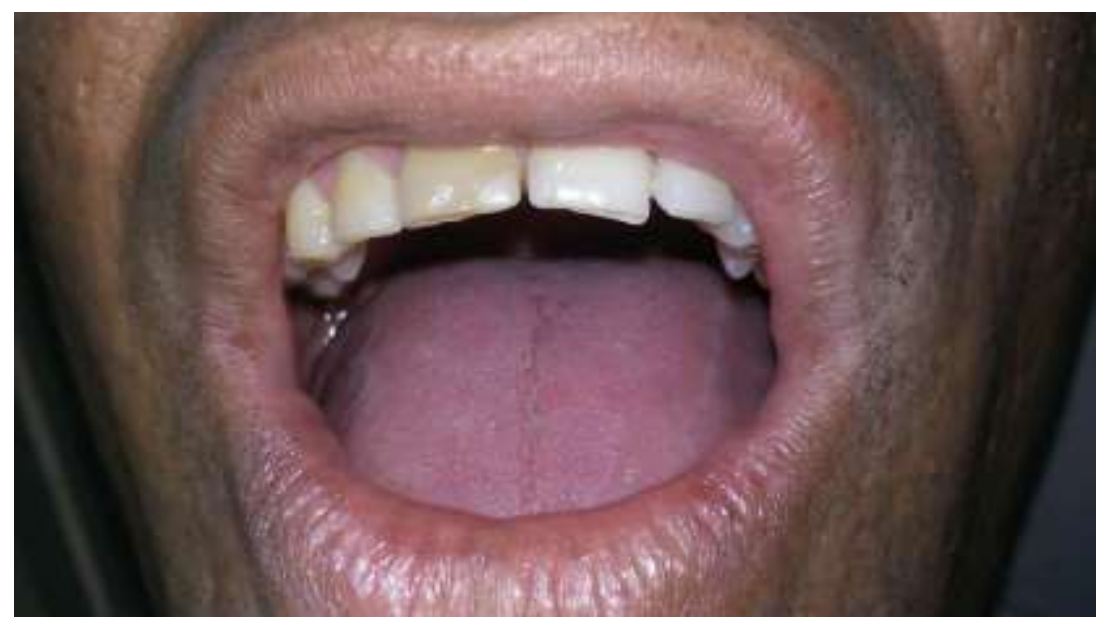

Fonte: Autores.

Figura 3 - Bruxismo em paciente do $1^{\circ} \mathrm{BPM}-\mathrm{PMAL}-2015$.

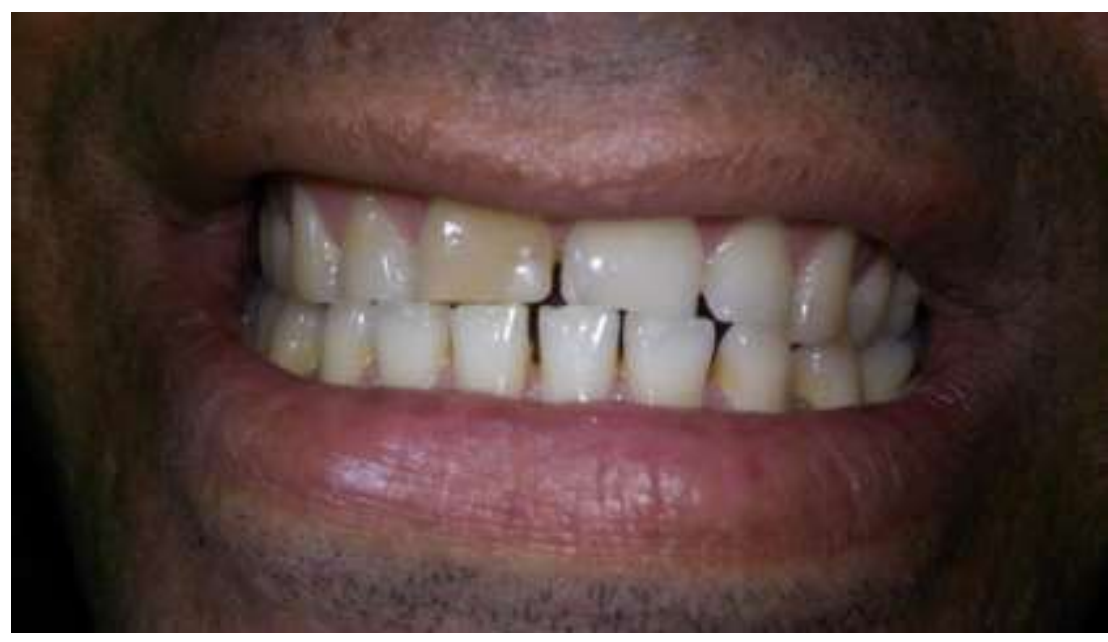

Fonte: Autores.

Na Figura 2 pode ser observado desgaste nas bordas incisais dos incisivos superiores em paciente com bruxismo. Na Figura 3, o mesmo paciente com as arcadas dentárias em oclusão.

O herpes labial foi observado clinicamente em um policial, encontrando-se na fase cicatricial (Figura 4). A prevalência relatada (8,92\% da amostra) foi um pouco abaixo do percentual descrito por Neville et al., (2004), que refere uma prevalência de herpes labial com variações entre $15-45 \%$ da população em geral. Porém, o resultado aproxima-se do apresentado por Mateus et al., (2006), no qual 9,38\% de uma amostra de estudantes de odontologia revelaram-se portadores da doença. Não houve diferenças significativas na prevalência com relação à idade e dos 10 casos encontrados apenas um aconteceu no gênero feminino. No estudo de Mateus et al., (2009), cujos pacientes apresentaram a idade variando entre 16 e 29 anos, ocorreu uma maior prevalência do herpes labial entre 21 e 24 anos, e as mulheres foram mais afetadas que os homens. Novamente a minoria percentual de mulheres no universo avaliado não permite uma correlação expressiva da prevalência da doença com o gênero. 
Figura 4 - Herpes labial (fase cicatricial) em paciente do $1^{\circ} \mathrm{BPM}$ - PMAL - 2015.

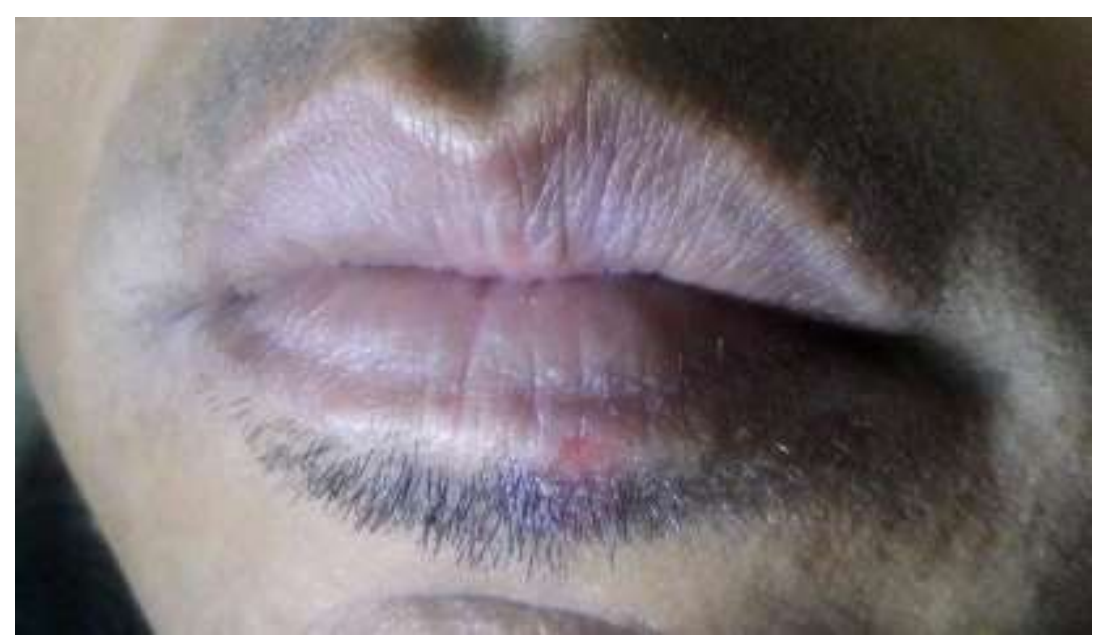

Fonte: Autores.

A xerostomia (sensação de boca seca) foi referida por $8,92 \%$ da amostra. Dos dez casos encontrados, apenas quatro utilizavam medicação que poderia contribuir para a xerostomia. Além do mais, os seis casos restantes apresentaram algum tipo de doença psicossomática (herpes labial, ulceração aftosa recorrente ou mucosa mordiscata). A análise estatística revelou associação entre a sensação de boca seca e diagnóstico de alguma doença psicossomática (p.=0,000 pelo teste exato de Fisher). Segundo Orellana et al., (2006), citados por Scarabelot (2010, p. 2) e Neville et al., (2004) a prevalência da xerostomia na população está em torno de $20 \%$, sendo mais comum em idosos. Não foi possível, devido às limitações da pesquisa, avaliar a redução do fluxo salivar, sendo os dados encontrados subjetivos, baseados nos relatos dos pacientes.

A mucosa mordiscata foi uma lesão relativamente frequente, correspondendo a 4,46\% da amostra (apresentando-se como na Figura 5 e Figura 6). Para Neville et al., (2004) 1 a cada 800 adultos possuem lesões ativas.

Figura 5 - Mucosa mordiscata em paciente do $1^{\circ} \mathrm{BPM}$ - PMAL - 2015.

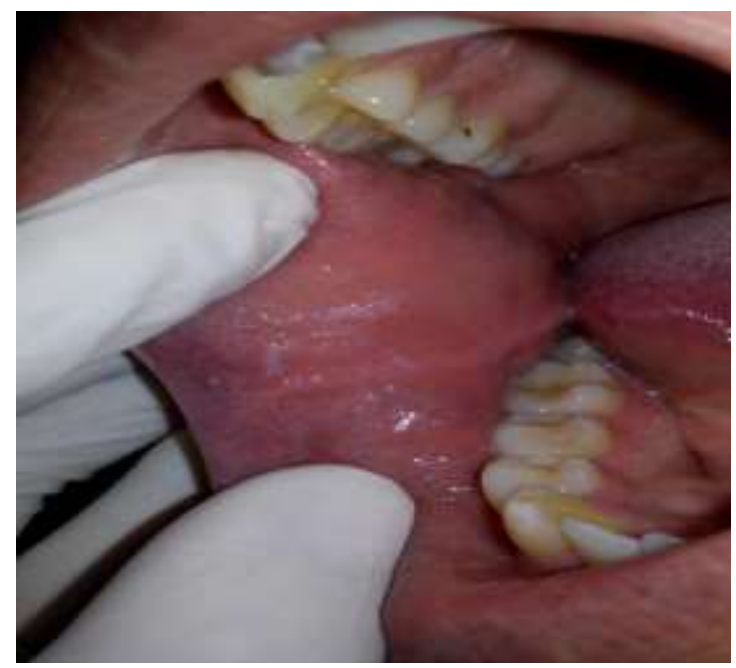

Fonte: Autores.

A Figura 5 expõe um caso de mordiscada crônica em mucosa jugal anterior, envolvendo áreas brancas, espessas e fragmentadas associadas a zonas eritematosas. A linha alba apresenta-se bastante evidenciada. 
Figura 6 - Mucosa mordiscata em paciente do $1^{\circ} \mathrm{BPM}$ - PMAL - 2015.

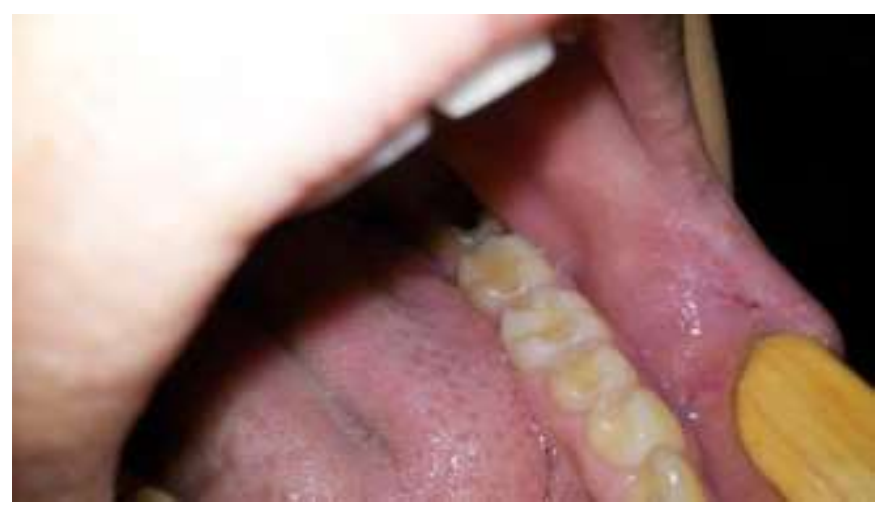

Fonte: Autores.

Pode-se visualizar na Figura 6 lesão mordiscada ulcerada próximo à comissura labial.

A língua geográfica representou 2,67\% dos examinados, corroborando este percentual com o descrito por Neville et al., (2004) que relatou uma frequência em torno de 1-3\% da população. O autor afirma, ainda, que as mulheres são afetadas com mais frequência que os homens, em uma razão de 2:1. Na presente pesquisa, onde o universo é predominantemente masculino, foram encontrados dois casos em homens (como o da Figura 7) e um caso em mulher.

Figura 7 - Língua geográfica em paciente do $1^{\circ}$ BPM - PMAL - 2015.

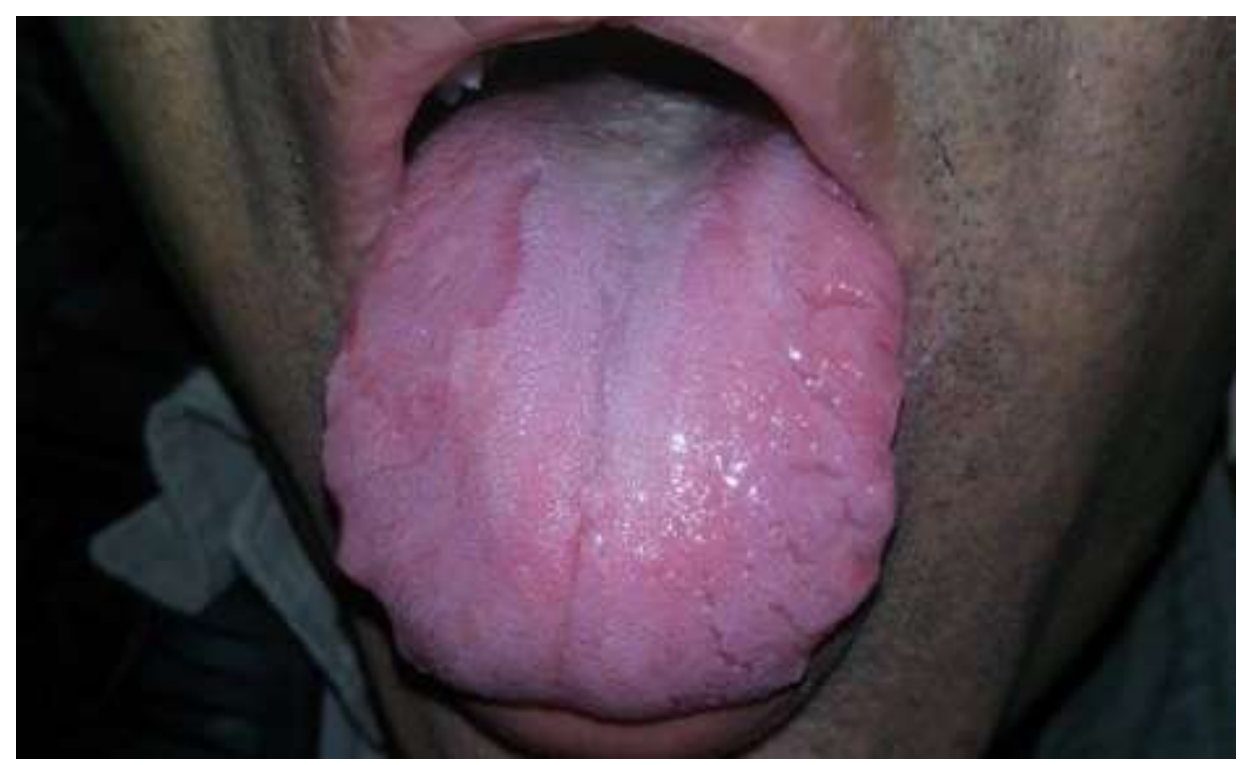

Fonte: Autores.

A Figura 7 apresenta o aspecto da língua geográfica, com áreas irregulares de perda de papilas filiformes, associada a outra condição bucal conhecida como língua fissurada.

Não foi observado nenhum caso de líquen plano na amostra avaliada. O fato de haver uma predominância do gênero masculino pode ter contribuído para a ausência da lesão, pois a prevalência desta em mulheres acontece em uma proporção de 3:2 em relação aos homens, além de abranger em torno de 0,1- 2,2\% da população, conforme afirma Neville et al., (2004).

Da mesma forma, não foi detectada a síndrome da boca ardente, mais frequente em mulheres após a menopausa.

Não foi possível, devido às limitações da pesquisa, a aplicação de testes psicológicos para avaliação do estresse. No entanto, foram considerados os resultados de um estudo anterior realizado pelo Centro de Assistência Social da PMAL (Alagoas, 
2012). De acordo com este estudo, $90 \%$ dos oficiais e $77 \%$ dos praças do $1^{\circ}$ BPM apresentaram alto nível de vulnerabilidade ao estresse no trabalho.

\section{Considerações Finais}

Foram detectadas as seguintes condições psicossomáticas: bruxismo, herpes labial, língua geográfica, mucosa mordiscata, ulceração aftosa recorrente e xerostomia. A ulceração aftosa recorrente foi a lesão mais encontrada. Observou-se uma relação estatística entre a xerostomia e o diagnóstico simultâneo de outras doenças psicossomáticas. A mucosa mordiscata foi uma condição relativamente frequente.

A prevalência de lesões bucais psicossomáticas nos pacientes avaliados representou, de uma forma geral, um padrão aproximado ao que é encontrado na população. Então, o estudo demonstrou que não ocorreu uma prevalência maior de lesões neste grupo, como se poderia imaginar, considerando-se que a pesquisa incluiu policiais pertencentes a um batalhão em que foi anteriormente constatado um alto nível de vulnerabilidade ao estresse. O fato de outros fatores, além do estresse, estarem envolvidos na etiopatogenia das referidas condições pode ter instigado a este resultado.

A pesquisa constituiu, ao mesmo tempo, uma forma de atenção à saúde do policial, visto que houve diagnóstico de lesões e orientações a respeito das mesmas.

Sendo este estudo considerado inicial (piloto) acerca da prevalência de lesões psicossomáticas em boca de militares da PMAL, propõe-se uma continuidade do mesmo nos demais batalhões ou, ainda, em outro grupo populacional, a fim de se aprofundar no tema proposto e para beneficiar um maior quantitativo de pacientes que seriam devidamente diagnosticados e orientados com relação a estas condições.

\section{Referências}

Alves, V. L.P. \& Lima, D. L. (2016). Percepção e enfrentamento do psicossomático na relação médico-paciente. Psicologia e Pesquisa, 32 (3). 1-9.

Barbosa, J. S. S., Santana, M. G. R. \& Macedo, S. (2020). Sintomas Psicossomáticos e stresse no trabalho de bombeiros militares: tecendo relações. Lumen, 29 (1). 09-26.

Carvalho, S. C. A., Carvalho, A. L. A., Lucena, S. C., Coelho, J. P. S. \& Araújo, T. P. B. (2008). Associação entre bruxismo e estresse em policiais militares. Revista Odonto Ciência. 23 (2). 125-129.

Carvallho, M. L. (2016). Qualidade de vida no trabalho versus condições psicossomáticas advindas do mercado de trabalho. REGRADE, UNIVEM, 9 (1). 67-84. Costa, A. R. F. et al. (2015). Orientações Metodológicas para Produção de Trabalhos Acadêmicos. Edufal.

Cruz, M. C. F. N., Braga V. A. S., Garcia, J. G. F., Lopes, F. F. \& Maia E. C. S. (2008). Condições bucais relacionadas com o estresse: uma revisão dos achados atuais. Revista da Faculdade de Odontologia de Porto Alegre. 49 (1). 8-11.

Estado de Alagoas, Secretaria de Estado da Defesa Social, Polícia Militar de Alagoas, Centro de Assistência Social. (2012). Relatório da pesquisa: Estresse e qualidade de vida no trabalho dos Policiais Militares de Alagoas - unidade

Kignel, S. et al. (2013). Estomatologia: Bases do Diagnóstico para o Clínico Geral. Santos.

Mateus, M. J. R., Duarte, I. L., Gondim, V. M. L. \& Sobral A. P. V. (2006). Avaliação do nível de estresse e sua correlação com o surgimento do herpes labial recorrente. Revista Brasileira de Pesquisa em Saúde. 19 (1). 35-39.

Neville, B. W., Damm, D. D., Allen, C. M. \& Bouquot, J. E. (2009). Patologia Oral e Maxilofacial. Guanabara Koogan

Pelegrini, A., Cardoso T. E., Claumann G. S., Pinto A. A. \& Felden E. P. G. (2018). Percepção das condições de trabalho e estresse ocupacional em policiais civis e militares de unidades de operações especiais. Cad. Bras. Ter. Ocup, 26 (2). 423-430.

Rabelo, L. D. B. C., Silva, J. M. A. \& Lima, M. E. A. (2018). Trabalho e adoecimento psicossomático: reflexões sobre o problema do nexo causal. Psicologia: Ciência e Profissão, 38 (1). 116-128.

Reche, R., Gomes, M. S., Pinto, J. N. \& Dick N. R. M. (2018). Associação entre bruxismo e a qualidade do sono em policiais militares. Revista saúde e desenvolvimento humano, 6 (1). 1-14.

Rangel, F. B. (2009). Sintomas Psicossomáticos e a Organização do Trabalho: um estudo em uma IES. XXXIII Encontro da ANPAD.

Reis, K. S. \& Godinho L. B. R. (2018). Psicossomática: uma visão holística do homem. Revista Cippus - Unilasalle, 6 (1). 1-14. 
Research, Society and Development, v. 10, n. 5, e22710514830, 2021

(CC BY 4.0) | ISSN 2525-3409 | DOI: http://dx.doi.org/10.33448/rsd-v10i5.14830

Sarranzi, H. C. \& Maia, P. R.M. (2020). Disfunção Temporomandibular e hábitos parafuncionais em policiais militares: um estudo transversal. Arq Odontol, 56 (21). 1-10.

Santos, R. O. B., Hauer, R. D. \& Furtado T. M. G. (2019). O sofrimento psíquico de policiais militares em decorrência de sua profissão. Revista Gestão e Saúde, 20 (2). 14-27.

Scarabelot, V. L. (2010). Análise de Fatores Psicológicos e Sistêmicos Associados à Queixa de Xerostomia. Dissertação (Mestrado em Ciências Médicas) Universidade Federal do Rio Grande do Sul.

Silva, O. J., Ferreira S. K. A., Silva S. F., Bergamini, G. B. Samuelsson, E,, Joner, C., Schneider L. F. \& Menz P. R. (2017). A correlação existente entre o estresse no ambiente de trabalho e doenças psicossomáticas. Revista científica da Faculdade de Educação e o Meio Ambiente, 8 (2). $2179-4200$.

Zanelli, J. C. et. al. (2010). Estresse nas Organizações de Trabalho compreensão e intervenção baseadas em evidências. Artmed. 